\title{
Roles of Reactive Oxygen Species and Mitochondria in Seed Germination
}

\author{
Muhammad Awais Farooq ${ }^{1,2 t}$, Xiaomeng Zhang ${ }^{1+}$, Muhammad Mubashar Zafar ${ }^{3 t}$, \\ Wei Ma ${ }^{1 *}$ and Jianjun Zhao ${ }^{1 *}$ \\ ${ }^{1}$ State Key Laboratory of North China Crop Improvement and Regulation, Key Laboratory of Vegetable Germplasm \\ Innovation and Utilization of Hebei, Collaborative Innovation Center of Vegetable Industry in Hebei, College of Horticulture, \\ Hebei Agricultural University, Baoding, China, ${ }^{2}$ Department of Plant Breeding and Genetics, University of Agriculture, \\ Faisalabad, Pakistan, ${ }^{3}$ Institute of Cotton Research, Chinese Academy of Agricultural Sciences, Anyang, China
}

\section{OPEN ACCESS}

Edited by: John Hancock,

University of the West of England, United Kingdom

Reviewed by: Alla l. Yemets,

National Academy of Sciences of Ukraine (NAN Ukraine), Ukraine Alma Balestrazzi, University of Pavia, Italy

*Correspondence: Wei Ma mawei0720@163.com Jianjun Zhao

jjz1971@aliyun.com

${ }^{\dagger}$ These authors have contributed equally to this work

Specialty section: This article was submitted to Plant Physiology, a section of the journal Frontiers in Plant Science

Received: 23 September 2021 Accepted: 18 November 2021 Published: 09 December 2021

Citation:

Faroog MA, Zhang X, Zafar MM, Ma W and Zhao J (2021) Roles of Reactive Oxygen Species and Mitochondria in Seed

Germination.

Front. Plant Sci. 12:781734. doi: 10.3389/fp/s.2021.781734
Seed germination is crucial for the life cycle of plants and maximum crop production. This critical developmental step is regulated by diverse endogenous [hormones, reactive oxygen species (ROS)] and exogenous (light, temperature) factors. Reactive oxygen species promote the release of seed dormancy by biomolecules oxidation, testa weakening and endosperm decay. Reactive oxygen species modulate metabolic and hormone signaling pathways that induce and maintain seed dormancy and germination. Endosperm provides nutrients and senses environmental signals to regulate the growth of the embryo by secreting timely signals. The growing energy demand of the developing embryo and endosperm is fulfilled by functional mitochondria. Mitochondrial matrixlocalized heat shock protein GhHSP24.7 controls seed germination in a temperaturedependent manner. In this review, we summarize comprehensive view of biochemical and molecular mechanisms, which coordinately control seed germination. We also discuss that the accurate and optimized coordination of ROS, mitochondria, heat shock proteins is required to permit testa rupture and subsequent germination.

Keywords: seed germination and dormancy, reactive oxygen species (ROS), mitochondria, heat shock proteins (HSPs), embryogenesis and endosperm

\section{HIGHLIGHTS}

- ROS inside cell due to external environmental dynamics signals seed germination.

- Mitochondria produce large amounts of ATP for seed germination.

- HSPs induces testa weakening and micropylar endosperm decay to release dormancy.

\section{INTRODUCTION}

Seed germination is crucial for uniform and maximum crop production (Carrera-Castaño et al., 2020), and the optimization of intrinsic and extrinsic factors is very important for promoting the

Abbreviations: ABA, Abscisic acid; GA, Gibberellins; PCD, Programmed cell death; ROS, Reactive oxygen species; ET, Ethylene; DOG, Delay of Germination; QTL, Quantitative trait loci; ME, Micropylar endosperm; TCA, Tricarboxylic acid cycle; HAI, Hours after imbibition; PPRs, Pentatricopeptide repeat proteins; HSP, Heat shock protein; PFE, Puncture force of endosperm; NOX, NADPH oxidase. 
transition from dormancy to germination (Figure 1) (Wang et al., 2020). The dormancy level of commercial crop varieties is typically lower than that of their wild relatives, as this helps to achieve maximum production. Nonetheless, the level of dormancy must be calibrated to prevent pre-harvest sprouting and consequent losses of yield and quality (Shu et al., 2016). A deeper understanding of seed germination and dormancy is therefore important for both agronomic and economic reasons.

The transition from seed dormancy to germination is a physiological process that is regulated by diverse endogenous hormones and environmental factors. ABA (abscisic acid) and GAs (gibberellins) are the main hormones that work in coordination to release seed dormancy and enable seed germination (Wang et al., 2019). Recent findings have revealed that auxins also play an important role in maintaining seed dormancy until conditions are favorable for plant survival (Shuai et al., 2017). Besides intrinsic cues, numerous extrinsic factors can prolong or terminate seed dormancy and promote seed germination and development. Light (Oh et al., 2004), temperature (He et al., 2016), and soil conditions (Meng et al., 2017) are major signals that can be perceived by seeds to regulate the timing of germination. The regulatory effect of light on seed germination depends on its spectrum, the red light increases the seed germination than any other light color (Stawska and Oracz, 2019).

Reactive oxygen species (ROS) have traditionally been viewed as destructive agents in plants; however, it has been recently explained that ROS also play a positive role in seed germination (Oracz and Karpiński, 2016). Oxygen can be transformed into multiple ROS, including singlet oxygen, superoxide, the hydroxyl radical, and hydrogen peroxide. Hydrogen peroxide is considered to be an important ROS agent, as it can pass readily through various cellular membranes (Anand et al., 2019). Reactive oxygen species production inside the seed transforms it from the quiescent seed produced by the mother plant into a biologically active seed that is capable of germination (Xia et al., 2018). This process occurs when environmental signals are accurately perceived and processed into endogenous signals by the seed (Bailly, 2019). The mitochondrion is known to carry out diverse functions in the cellular landscape. It synthesizes vitamins, (i.e., ascorbic acid, folic acid, and biotin) and selected amino acids (Foyer et al., 2020), and has a pivotal role in programmed cell death (PCD) (Zhao et al., 2018). Beside a major site for ROS production, mitochondrion also plays a significant role in seed germination (Bailly, 2019). Heat shock proteins localized in the mitochondria play a crucial role in seed germination through temperature-dependent ROS generation (Ma et al., 2019). This article mainly summarizes the roles of ROS, heat shock proteins and mitochondria in seed germination.

\section{ROLE OF REACTIVE OXYGEN SPECIES IN SEED GERMINATION}

Reactive oxygen species (ROS) are produced in the seed as a result of metabolism and play a significant role in seed germination (Table 1). Weakening of the endosperm is a prerequisite for the initiation of seed germination and is driven by various internal and external factors. The decay of the endosperm is directly linked to the production of ROS in response to the availability of external environmental signals (Zhang et al., 2019). ROS include free radicals such as singlet oxygen $\left({ }^{1} \mathrm{O}^{-2}\right)$, superoxide $\left(\mathrm{O}^{-2}\right)$ or the hydroxyl radical $(\mathrm{OH})$ (Bailly, 2019). Hydrogen peroxide is a reactive molecule that performs a signaling function in seed germination and has the ability to cross biological membranes (Hajihashemi et al., 2020). During the germination of the seed, the endogenous $\mathrm{H}_{2} \mathrm{O}_{2}$ accumulates inside the seed so the germination can occur, and seed coat can loosen up. When the production of $\mathrm{H}_{2} \mathrm{O}_{2}$ will begin to damage the internal organelles, the defense mechanisms inside the cell will be activated. Recently hydrogen peroxide responsive genes $(H R G) 1$ and 2 have been identified that play a significant role in containing the production of $\mathrm{H}_{2} \mathrm{O}_{2}$ inside the cell so that plant metabolic process cannot be disrupted (Gong et al., 2021). The specialty of HRG1 and HRG2 is that they will keep rather low content of protein until the level of $\mathrm{H}_{2} \mathrm{O}_{2}$ is elevated, which discerns them from the other hydrogen peroxide responsive genes providing novel pathway for $\mathrm{H}_{2} \mathrm{O}_{2}$ sensing and response. During the germination process, this novel pathway is involved in the meristem activity regulation of embryonic roots. The novel HRG1 and HRG2 genes express to protect the microenvironmental stability of root tip meristem for maintain meristem cell normal activity concerning both cell division and elongation (Gong et al., 2021). Abiotic and biotic stresses can result in oxidative stress that causes programmed cell death. In addition to their negative effects, ROS also play a significant positive role in dormancy release, seed germination signaling, protection against pathogens, and regulation of internal cellular machinery in response to external environmental dynamics. There is a specific "oxidative window" that allows cellular events to unfold in sequential order for seed germination if ROS are maintained within a particular range (Anand et al., 2019). To date, ROS dynamics have been studied mainly after water imbibition because they are easier to measure at this time.

After ripening, seeds are quiescent and very low in moisture. Measurement of ROS production and metabolism in dry seeds is very challenging, mainly due to technical barriers. Oxygen is the main driver for ROS production in anhydrobiotic seeds. It typically exists in its ground state $\left({ }^{3} \mathrm{O}_{2}\right)$ with two unpaired electrons with parallel spins, and its reduction generates various forms of ROS. Oxygen is present primarily in the void spaces of dry seeds, and these dry spaces collectively form an air space network (Ahmed et al., 2018). Seeds are desiccated on the mother plant after ripening, but mother plants generate ROS to create an oxidative environment inside the seed. During seed storage, available oxygen kickstarts chemical reactions. Lipids can be easily oxidized in seeds under low moisture conditions and therefore serve as a source of free radicals. The increase in water uptake in zone 1 of the water sorption isotherm reduces lipid oxidation by filling the pore spaces and decreasing the oxygen concentration inside the seed, resulting in a low reaction rate. Lipid peroxidation in the low moisture system may lead to the reactions of proteins with lipid hydroperoxides, free radicals, and peroxide breakdown products (Cai et al., 2011). The key function of oxygen in the seed is the release of seed dormancy, as has been 


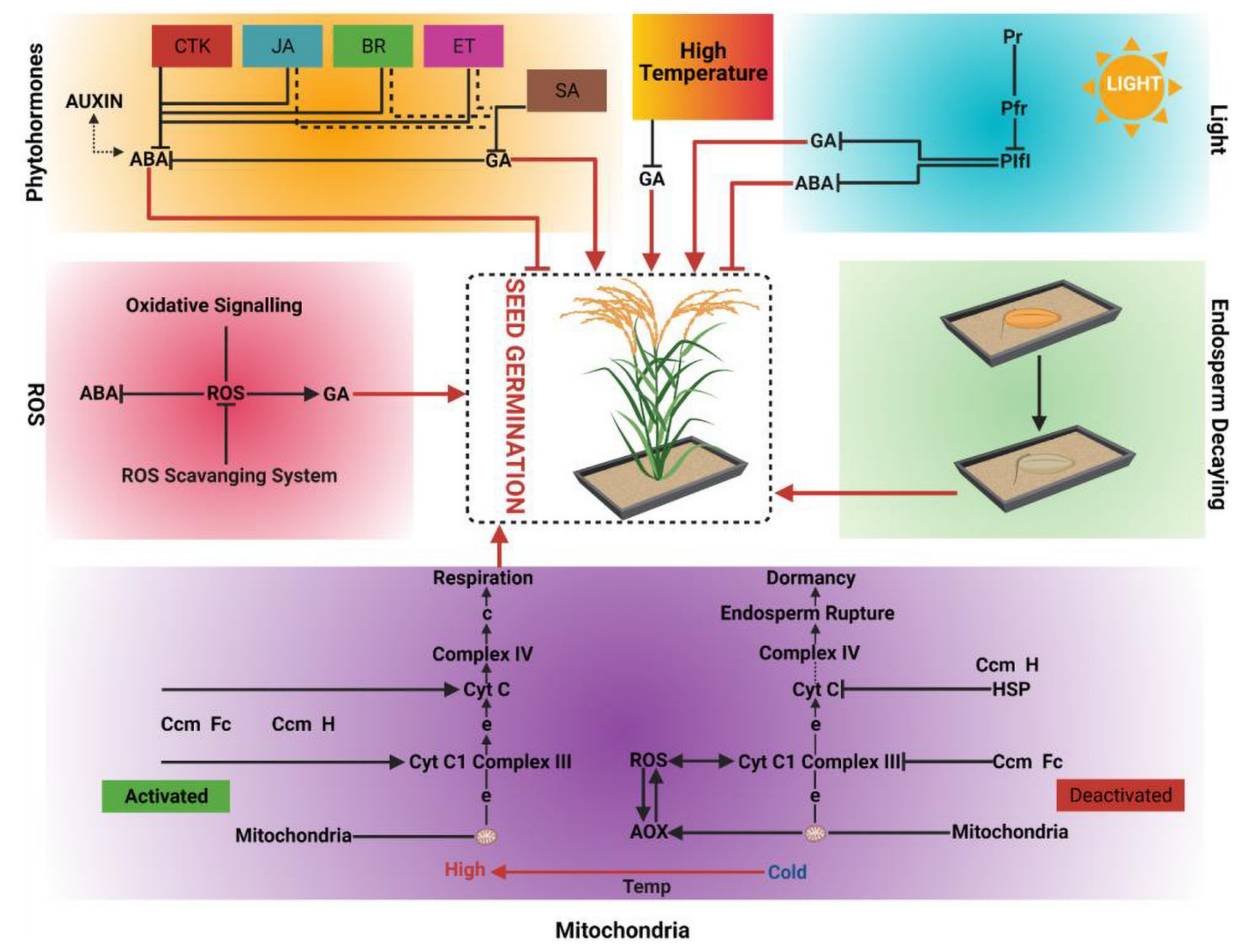

FIGURE 1 | Driving forces of seed germination; Phytohormones, High temperature, Light, Reactive oxygen species (ROS), Endosperm decaying and Mitochondria. CTK: Cytokinins, JA: Jasmonic acid, BR: Brassinosteroids, ET: Ethylene, SA: Salicylic acid, GA: Gibberellic acid, ABA: Abscisic acid, PIF1: Phytochrome Integrating Factor 1, Pfr: Photoreceptors far red, Pr: Photoreceptor red, Cyt c: Cytochrome c, AOX: Alternative oxidases, HSP: Heat shock proteins, ROS: Reactive oxygen species, Temp: Temperature.

observed in experiments with barley, Arabidopsis, and sunflower (Leymarie et al., 2012; Beracochea et al., 2015).

The resumption of metabolic activity in the seed is related to the regulated enzymatic production of ROS. Seeds are very sensitive to water, which leads to the production of ROS. The decision to germinate is dependent on various environmental stimuli such as temperature, moisture, and light (Gomes and Garcia, 2013). Major sites involved in the production of ROS are the mitochondria, the peroxisome, and the NADPH oxidases of the plasma membrane. The inhibition of NADPH oxidase delays seed germination, underscoring the important function of this enzyme. After imbibition, the resurgence of mitochondrial respiration in the seed may cause electrons to be donated to oxygen as an electron acceptor, leading to ROS production (Kranner et al., 2010). NADPH oxidases, also known as respiratory burst oxidase homologs (Rbohs) after the homology with gp $91^{\text {phox }}$ domain of the mammalian respiratory burst oxidase are main drivers of ROS production in the cell wall space which leads to seed germination through nonenzymatic way and results in radicle elongation and endosperm cap weakening in dicots (Sun L. R. et al., 2019). Rbohs transfers the electron from NADPH or NADH to apoplastic oxygen which results in the production of superoxide radicals and these radicals can directly cleave polysaccharides which will loosens the plant cell walls (Yang et al., 2020). As a typical dicot seed, i.e., Lactuca sativa has two layers of seed coverings, a dead and soft seed coat and an endosperm tissue covered with 2-3 layers of the living cells (Jones, 1974). NADPH oxidases as non-enzymatic mode along with enzymatic mechanisms, i.e., pectin-methyltransferases, cellulase and hemicellulose degrading enzymes are involved in the loosening and degradation of these layers so that the germination can occur (Zhang et al., 2014). Protein carbonylation and protein turnover increase owing to the accumulation of $\mathrm{H}_{2} \mathrm{O}_{2}$. It also causes a decrease in electron pressure in the mitochondrial electron transport chain, allowing the provision of reducing equivalents (NADPH) to the thioredoxin (Trx) system (via the pentose phosphate pathway), which is involved in the regulation of seed germination and seedling development (Li et al., 2017). The $\mathrm{H}_{2} \mathrm{O}_{2}$ concentration also affects the hormone balance by increasing GA and decreasing ABA/ethylene via 1-aminocyclopropane-1-carboxylic acid. This remodeling of hormone signaling may lead to the recommencement of metabolic activity that is essential for seed germination and seedling emergence (El-Maarouf-Bouteau and Bailly, 2008).

In monocots, the coleorhiza, a non-vascularized multicellular embryonic tissue, covers the seminal roots of monocot seeds and 
TABLE 1 | Effects of ROS on seed germination in various plant species.

\begin{tabular}{|c|c|c|c|}
\hline Context & Effect & Species & References \\
\hline Dormancy alleviation & Positive & Malus domestica & Dębska et al., 2013 \\
\hline Salt stress & Negative & Arabidopsis thaliana & Yang et al., 2019b \\
\hline ABA cross talk & $\begin{array}{l}\text { ABA positive regulator } \\
\text { of rboh and ROS }\end{array}$ & Arabidopsis thaliana & Yang et al., 2019a \\
\hline Mitochondrial functioning & Positive & Arabidopsis thaliana & Ma et al., 2019 \\
\hline Salt stress & Positive & Arabidopsis thaliana & Ortiz-Espín et al., 2017 \\
\hline Seed dormancy and iron deficiency & Positive & Arabidopsis thaliana & Murgia and Morandini, 2017 \\
\hline Germination/ABA & Negative & Arabidopsis thaliana & Baek et al., 2015 \\
\hline Salt stress/ethylene & Negative & Arabidopsis thaliana & Lin et al., 2013 \\
\hline Germination/ABA signaling & Positive & Arabidopsis thaliana & Bi et al., 2017 \\
\hline Dormancy/ABA/GA & Positive & Hordeum vulgare & Bahin et al., 2011 \\
\hline Seed germination and dormancy & Positive & Hordeum vulgare & Ma et al., 2017 \\
\hline Germination/ABA signaling & Positive & Hordeum vulgare & Ishibashi et al., 2017 \\
\hline Dormancy alleviation & Positive & Hordeum vulgare & Ma Z. et al., 2016 \\
\hline Germination/GA/NADPH oxidase & Positive & Hordeum vulgare & Kai et al., 2016 \\
\hline Germination/NADPH oxidase & Positive & Hordeum vulgare & Ishibashi et al., 2015 \\
\hline Dormancy & Positive & Bidens Pilosa & Whitaker et al., 2010 \\
\hline Dormancy alleviation & Positive & Bunium persicum & Amooaghaie and Ahmadi, 2017 \\
\hline Dormancy alleviation & Positive & Hedysarum scoparium & Su et al., 2016 \\
\hline Low phytic acid seed vigor & Positive & Oryza Sativa & Zhou et al., 2018 \\
\hline Dormancy alleviation (after ripening) & Positive & Oryza Sativa & Zhang et al., 2017 \\
\hline Germination NADPH oxidase & Positive & Oryza Sativa & Li et al., 2017 \\
\hline Osmotic and salt stress & Negative & Oryza Sativa & Chen et al., 2016 \\
\hline Germination/ABA/GA & Positive & Oryza Sativa & Ye and Zhang, 2012 \\
\hline Germination/ethylene & Positive & Sorghum bicolor & Ishibashi et al., 2013 \\
\hline Dormancy alleviation (after ripening) & Positive & Helianthus annus & Morscher et al., 2015 \\
\hline Dormancy alleviation/ABA/ethylene & Positive & Helianthus annus & El-Maarouf-Bouteau et al., 2015 \\
\hline Dormancy & Positive & Helianthus annus & Oracz et al., 2007 \\
\hline GA response & Positive & Nicotina tabacum & Oracz et al., 2009 \\
\hline Germination & Positive & Vigna radiata & Singh et al., 2015 \\
\hline Seed vigor and GA signaling & Positive & Citrullus lanatus & He et al., 2019 \\
\hline Dormancy & Positive & Triticum aestivum & Bykova et al., 2011 \\
\hline
\end{tabular}

is thought to have a role in protecting the emerging root and it is also involved in the regulation of radicle emergence upon the germination of monocots seeds, i.e., wheat (González-Calle et al., 2015). The embryo is located on the side of endosperm, where it is considered that endosperm is involved in providing the nutrition to the growing embryo. During germination process, the epiblast and coleorhiza appears first and then the primary leaf appears from the coleoptile (Betekhtin et al., 2018). The role of coleorhiza is known to protect the emerging roots during seed germination; however, it has been established that it plays the same role in monocots which micropylar endosperm plays in the dicots, i.e., it acts as a barrier for radicle protrusion to 
complete germination (sensu stricto) (Holloway et al., 2021). The dissolution of coleorhiza occurs after $6 \mathrm{~h}$ of imbibition of the water in rice and thus its degeneration is pre-requisite for the germination of the seeds (Barrero et al., 2009). The nonenzymatic mechanisms, i.e., the production of ROS is directly linked with the increase in the germination especially the accumulation and production of $\mathrm{O}^{2-}, \mathrm{H}_{2} \mathrm{O}_{2}$, and $\mathrm{OH}^{-}$radicals were higher in coleorhiza and radicle than in the coleoptile of germinating seeds ( $\mathrm{Li}$ et al., 2017). Moreover, NADPH oxidases (NOXs) are kind of proteins and are major enzymatic route of ROS synthesis in rice seed germination (Kai et al., 2016). The inhibition of NOXs in monocots can results in the delayed germination because these are involved in radicle and root growth. NOXs mRNAs are reported to express in the embryo and aleurone cells of barley seeds (Iglesias-Fernández et al., 2020), these expression sites are in conformity with the sites of ROS production in the seeds after imbibition (Ishibashi et al., 2015).

\section{MITOCHONDRIA ARE INDISPENSABLE FOR SEED GERMINATION}

The mitochondrion is responsible for regulating various functions in the cell. It is involved in the production of vitamins (ascorbic acid, folic acid, and biotin) (Foyer et al., 2020), and selected amino acids so that cellular processes are carried out uninterrupted (Wagner et al., 2018). Moreover, under abiotic and biotic stress, it causes programmed cell death to put plant in a better position to combat these stresses. Mitochondria also induces the production of ROS which functions in the signaling pathways of cellular networks. It also has key roles in diverse metabolic pathways such as iron homeostasis, lipid metabolism, and nitrogen assimilation. Its most established role is in the production of cellular energy in the form of ATP through oxidative phosphorylation (Law et al., 2014). The onset of germination is marked by the conversion of simple, quiescent promitochondria of dry mature seeds into energetic, metabolically active, cristae-containing organelles. The study of sunflower seeds by transmission electron microscopy has shown that low density of the mitochondrial matrix, absence of a discontinuous outer membrane, and few cristae are associated with low ATP production through oxidative phosphorylation (Czarna et al., 2016). In other crops, such as rice (Taylor et al., 2010), maize (Bahaji et al., 2019), and peas (Henriet et al., 2021), the presence of mature mitochondria with large numbers of continuous cristae structures, an electron dense matrix, and abundant electron transport chain components were suggestive of high protein contents that enable the seed to produce large amounts of ATP through increased metabolic activity and respiration. Isolated promitochondria from rice seeds were rich in proteins but were unable to be metabolically active without imbibition. When rice seed was imbibed for $30 \mathrm{~min}$, its mitochondrial metabolic and protein import functions were restored. Therefore, imbibition is a prerequisite for the transformation of promitochondria into mature and fully differentiated mitochondria. As seed germination proceeds, the import of components for mitochondrial function is reduced, and the import of machinery for primary metabolism in the cell increases (Best et al., 2020). The rate of protein import continues to rise, whereas the abundance of protein import machinery declines in the cell overall. This suggests that there may be some degradation of import machinery in the cell as the concentration of promitochondria decreases and that of mature mitochondria increases (Law et al., 2014).

\section{CHANGES IN THE MITOCHONDRIAL TRANSCRIPTOME DURING SEED GERMINATION}

Dynamics of mitochondrial genes changes quantitatively as the germination progresses which validates that mitochondrial transcription and in turn the production of proteins is very vital for the germination of seeds. Northern blot analysis in maize showed that transcripts of genes associated with mitochondrial biogenesis such as atpa, atp9, $\operatorname{cox} 1, \operatorname{cox} 2$, and $\operatorname{cox} 3$ were present at low abundance during early time points of seed germination but increased in abundance from 24 to $48 \mathrm{~h}$ after imbibition (HAI) (Logan et al., 2001). Emp12 encodes a P-subfamily PPR protein that is located in the mitochondria (Small et al., 2004) and its expression is ubiquitous in a range of vegetative and reproductive tissues, which is mostly involved in the development of kernels (Cheng et al., 2016). The maize Emp12 is involved in embryogenesis and endosperm development, the mutation in Emp12 restricts the embryo and endosperm development causing embryo fatality. At the 16 DAP the kernels which were Emp12 mutated were much smaller in size and microscopy study indicated that this mutation resulted in crumbled empty pericarp, accumulated less starch and the embryo development halted at the transitional stage, rendering an undifferentiated embryo (Sun F. et al., 2019). It suggests that mitochondrial localized Emp12 plays an essential role in the embryogenesis and endosperm development. Moreover, the Emp12 mutation causes trans-splicing of mitochondrial nad2 intron 2 and cissplicing of nad2 intron 4 are obstructed, whereas the cis-splicing of nad2 intron 1 is reduced in Emp12 mutants (Cheng et al., 2016; Dai et al., 2018). It results in the dismantling of the complex1 assembly, and its activity decreases manifolds in the mutants and the expression of other alternative oxidases, and several other mitochondrial complexes greatly increases (Xiu et al., 2016; Qi et al., 2017). Therefore, Emp12 is inevitable for the accurate and timely trans-splicing of nad2 intron 2 and cis splicing of nad2 introns 1 and 4 and plays a significant role in the complex 1 biogenesis, embryogenesis and endosperm development in maize (Sun F. et al., 2019).

Similarly, a study in rice demonstrated that there was a low concentration of mitochondrial transcripts 3 HAI and a peak in transcripts related to mitochondrial energy production and metabolism at 12 HAI (Howell et al., 2009). Transcriptome data indicated that a small surge in transcripts in the germinating seed at 3 HAI was specifically associated with mitochondrial proteins rather than any other organelle, suggesting that the activation of mitochondria is essential for seed germination after imbibition (Logan et al., 2001). The analysis of genes that encode mitochondrial proteins has demonstrated a triphasic progression of transcriptomic events during seed 
germination (Law et al., 2012). Initially, two groups of genes are transiently expressed. The first group shows the highest transcript abundance until the end of stratification and before the seed is exposed to continuous light. They encode proteins that function in nucleic acid metabolism. The second group encodes proteins with import and synthesis functions. They are followed by a third group of genes that encode electron transport chain components and whose transcript abundance progressively increases. During the first transcriptomic phase, the first two groups of genes are activated, and the abundance of proteins related to DNA and RNA metabolism increases. These include proteins that function in transcription, RNA editing, splicing, processing, stability, and translation. Specifically, there is over representation of mitochondrial targeted pentatricopeptide repeat proteins (PPRs). PPRs are mitochondria or plastid-targeted proteins with diverse functions in RNA metabolism: RNA editing (ChateignerBoutin et al., 2008), transcription (Ding et al., 2006), splicing (De Longevialle et al., 2008), processing (Nakamura et al., 2003), stability (Yamazaki et al., 2004), and translation (Choquet and Wollman, 2002). The expression of these transcripts is synchronized with the expression of transcripts encoding proteins that function in cytosolic nucleotide metabolism and factors responsible for conveying these nucleotides into the mitochondrial matrix. This pattern of transcription suggests that the coordinated production and transport of nucleotides is important, enabling them to be used for the transcription of mitochondrial genes (Li et al., 2012). During the second phase, the second group of genes is activated, including transcripts that encode ribosomes, translation factors, and tRNA-related functions (Narsai et al., 2011). There is an increased abundance of proteins related to mitochondrial protein import, including components of the inner membrane (TIM) and the outer membrane (TOM) complexes. This suggests that the import of nuclear-encoded mitochondrial proteins goes hand-in-hand with the synthesis of organelle-encoded proteins. This is necessary because many mitochondrial protein complexes contain both nuclear- and organelle-encoded subunits, and their successful assembly requires careful control of subunit accumulation (Jänsch et al., 1996). The presence of these biogenesis factors early in the germination time course highlights the significance of mitochondrial biogenesis for successful seed germination (Law et al., 2012). The third group of mitochondrial transcripts encodes components of the electron transport chain. The abundance of these proteins increases as germination progresses and it peaks before the end of germination. This marks the third and final phase in the transition of promitochondria to mature mitochondria and establishes the metabolic functions that are required for the vegetative stages of plant development (Figure 2) (Howell et al., 2009).

\section{CHANGES IN THE MITOCHONDRIAL PROTEOME DURING SEED GERMINATION}

Numerous mitochondrial proteins are involved in seed germination; they perform various functions such as respiration, metabolism, import or transport, carbon metabolism/tricarboxylic acid cycle (TCA) reactions, stress responses, and chaperone activities. The abundance of these proteins in seed mitochondria at the time of germination indicates that they are indispensable for seed germination (Hao et al., 2019). Galland performed a proteome study in the mitochondria of Arabidopsis seeds within the first 0-24 h of germination (Galland et al., 2014). Two hundred fifty-seven non-redundant proteins from 475 identified mitochondrial protein spots showed various responses during seed germination. There were mitochondrial proteins that were up- or down regulated or remained at a constant level during the seed germination. The mitochondrial proteins which were upregulated were glutamate dehydrogenase 1 or 3 , monodehydroascorbate reductase, glyceraldehyde-3-phosphate dehydrogenase, succinate-semialdehyde dehydrogenase, the beta subunit of ATP synthase, aconitate hydratase 3, phosphoenolpyruvate carboxykinase, the beta subunit of mitochondrial processing peptidase, Hsp 60, and translation elongation EF-Tu. The proteins which remained at constant levels from 0-24 h, including succinyl-CoA ligase alpha-chain, the flavoprotein subunit of succinate dehydrogenase, and Hsp 70-2. A few newly synthesized proteins decreased in abundance over the 24-h germination period, such as the alpha subunit of ATP synthase and superoxide dismutase 2 (Czarna et al., 2016). Some proteins that were not newly synthesized but decreased over the germination period included the NADH-ubiquinone oxidoreductase $75 \mathrm{kDa}$ subunit, superoxide dismutase 1 , and late embryogenesis abundant proteins. The relative abundance of specific proteins at different time points and selective mRNA translation highlight those proteins that are important for the process of seed germination (Rao et al., 2017).

\section{HEAT SHOCK PROTEINS ARE THE ULTIMATE DECISION-MAKERS IN SEED GERMINATION}

In addition to their established role in protection from heat, heat shock proteins play a significant part in seed germination and development (Kaur et al., 2016). Seed germination depends on the surrounding temperature, which can delay or expedite the process after sowing (Ding et al., 2020). The germination efficiency of the cotton seed is related to warm temperatures in a narrow window ranging from $20^{\circ} \mathrm{C}$ to $36^{\circ} \mathrm{C}$ (Zhang et al., 2015; Ma W. et al., 2016). The functional characterization of NnHSP17.5 gene in sacred lotus (Nelumbo nucifera Gaertn) has revealed that heat shock proteins (HSPs) play an important role in the seed germination and in the protection of the seeds against heat stress (Zhou et al., 2012). NnHSP17.5 is a member of class 2 proteins which are localized in cytoplasm and nucleoplasm, and specifically expressed in seeds development at later stages under normal conditions and are strongly up regulated in the germinating seeds upon heat and oxidative stresses (Scharf et al., 2001). When the heat stress goes beyond $42^{\circ} \mathrm{C}$, the NnHSP 17.5 expression gets instantly upregulated and in case if there is high concentration of $\mathrm{H}_{2} \mathrm{O}_{2}$ causing the oxidative stresses then again the NnHSP17.5 expressions in the germinating seed increases 


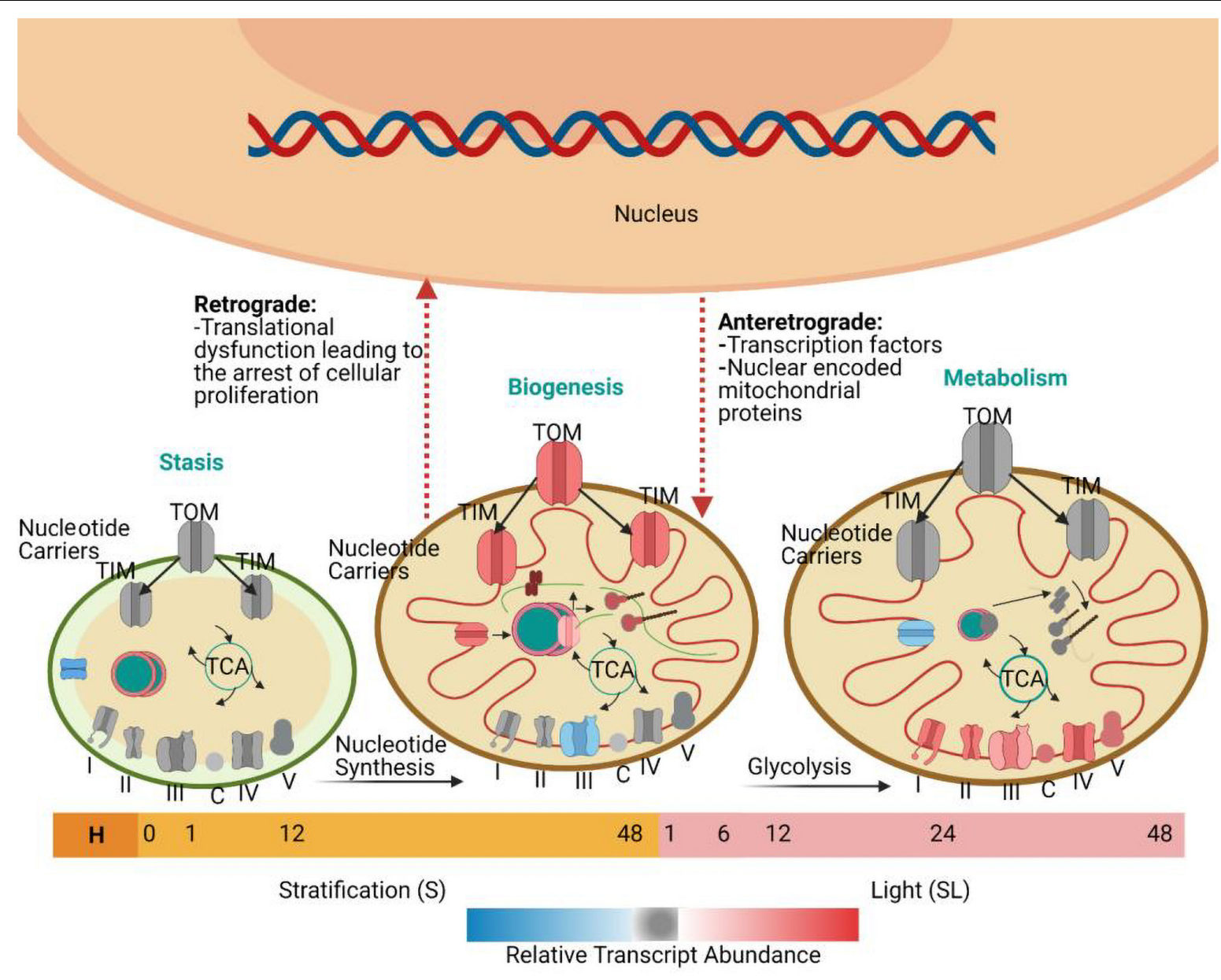

FIGURE 2 | Mitochondrial biogenesis during seed germination, based on the work of Law et al. (2012). Seeds were collected at ten time points: $\mathrm{H}$ (freshly harvested); $\mathrm{O}$ h (dry seeds after two weeks of desiccation); $1 \mathrm{~h} \mathrm{~S}, 12 \mathrm{~h} \mathrm{~S}$, and $48 \mathrm{~h} \mathrm{~S}$ [three time points during seed stratification (S)]; and $1 \mathrm{~h} \mathrm{SL}, 6 \mathrm{~h} \mathrm{SL}, 12 \mathrm{~h} \mathrm{SL}, 24 \mathrm{~h} \mathrm{SL}$, and $48 \mathrm{~h} \mathrm{SL}$ [five time points during the exposure to continuous light after stratification (SL)]. The promitochondria in dry seeds do not have the cristae that are associated with mature mitochondria. The transient expression of transcripts encoding proteins associated with DNA and RNA metabolism and nucleotide synthesis and import occurs when the seed is shifted from stratification to continuous light. Afterward, there is transient expression of transcripts encoding proteins for protein metabolism and import functions. During the next stage, after $24 \mathrm{~h}$ of continuous light, there is increased expression of genes encoding various metabolic components associated with the Tricarboxylic acid (TCA) cycle and the electron transport chain. The progress of each minute event in this process is closely monitored by the nucleus and mitochondrion through processes known as antegrade and retrograde regulation. TIM, the inner membrane; TOM, the outer membrane.

(Bailly et al., 2008). The role of small HSP17.4 as a regulator of thermotolerance during the seed development has shown in Arabidopsis thaliana seeds that the seeds which indicated high tolerance against desiccation had high concentration of sHSP17.4 during seed development in comparison to the mutants which were producing low concentration of sHSP17.4. Moreover, the study indicated that the seeds indicated low dormancy when the concentration of HSP17.4 was high or equal to the wild type in comparison to the mutant which expressed reduced concentration of HSP 17.4 (1\%-2\% of wild type) or at undetectable levels (Wehmeyer and Vierling, 2000).

Mitochondria I subfamily small heat shock proteins (msHSPs) are significantly induced during seed germination, especially GhHSP24.7 in cotton. There was a positive correlation $(R=0.99)$ between GhHSP24.7 expression levels and the change in temperature from $4^{\circ} \mathrm{C}$ to $36^{\circ} \mathrm{C}$, underscoring the importance of this HSP in seed germination. Likewise, GhHSP24.7 overexpression lines showed faster germination than those in which GhHSP24 expression was suppressed (Ma et al., 2019). This result confirmed that HSPs have a significant role in seed germination (di Donato and Geisler, 2019). Heat shock proteins regulate seed germination in response to temperature dynamics. For example, GhHSP24.7 accelerates seed germination by $50 \%$ when the temperature is increased from $20^{\circ} \mathrm{C}$ to $36^{\circ} \mathrm{C}$ (Ma et al., 2019). However, the germination of various crop plants begins to decline if temperatures increase above an optimum range. The germination percentage of wheat decreased when the temperature rose above $30^{\circ} \mathrm{C}$, and its germination percentage at $45^{\circ} \mathrm{C}$ was only $12 \%$ (Essemine et al., 2010).

Heat shock proteins (HSPs) also play a key role in the weakening of the testa so that the embryo can achieve its growth potential (Wehmeyer and Vierling, 2000). Seeds from 
GhHSP24.7 suppression lines had higher puncture force of endosperm (PFE) values than seeds with normal levels of GhHSP24.7. High PFE indicates a strong penetration resistance of the seed covering layers, and seeds in which GhHSP24.7 was suppressed showed both high PFE and delayed germination. ROS is a dominant factor in the transformation of the quiescent seed to a metabolically active organism, and GhHSP24.7 improves seed germination by increasing ROS production. Ma et al., observed that seeds with high levels of GhHSP24.7 expression underwent timely germination, whereas seeds with low GhHSP24.7 expression produced less $\mathrm{H}_{2} \mathrm{O}_{2}$ and $\mathrm{O}^{2-}$, which delayed germination (Ma et al., 2019). GhHSP24.7 also influenced the cellular structure of the seeds. Its high expression in seeds indicated that the two layers of cells in the endosperm were beginning to separate, marking the decay of cell structure in the micropylar endosperm. The function of HSPs is conserved in various plants such as tomato and Arabidopsis, as demonstrated by the presence of functional orthologs in the two genomes. Two Arabidopsis mitochondrial sHSPs (AtHSP23.5 and AtHSP23.6) and two tomato mitochondrial sHSPs (SlHSP23.8B and SlHSP21.5B) are reported to have definite roles in seed germination (Ma et al., 2019).

In summary, seed dormancy and seed germination respond to an interplay of endogenous and exogenous factors (Liu and Hou, 2018).

\section{CONCLUSION}

Uniform seed germination is essential for maximizing crop production. Seed germination is a very complex process

\section{REFERENCES}

Ahmed, A. K. A., Shi, X., Hua, L., Manzueta, L., Qing, W., and Marhaba, T. (2018). Influences of air, oxygen, nitrogen, and carbon dioxide nanobubbles on seed germination and plant growth. J. Agric. Food Chem. 66, 5117-5124. doi: 10.1021/acs.jafc.8b00333

Amooaghaie, R., and Ahmadi, F. (2017). Triangular interplay between ROS, ABA and GA in dormancy alleviation of Bunium persicum seeds by cold stratification. Russ. J. Plant Physiol. 64, 588-599. doi: 10.1134/s1021443717040021

Anand, A., Kumari, A., Thakur, M., and Koul, A. (2019). Hydrogen peroxide signaling integrates with phytohormones during the germination of magnetoprimed tomato seeds. Sci. Rep. 9:8814. doi: 10.1038/s41598-01945102-5

Baek, D., Cha, J.-Y., Kang, S., Park, B., Lee, H.-J., and Hong, H. (2015). The Arabidopsis a zinc finger domain protein ARS1 is essential for seed germination and ROS homeostasis in response to ABA and oxidative stress. Front. Plant Sci. 6:963. doi: 10.3389/fpls.2015.00963

Bahaji, A., Muñoz, F. J., Seguí-Simarro, J. M., Camacho-Fernández, C., RivasSendra, A., and Parra-Vega, V. (2019). Mitochondrial Zea mays Brittle1-1 is a major determinant of the metabolic fate of incoming sucrose and mitochondrial function in developing maize endosperms. Front. Plant. Sci. 10:242. doi: 10. 3389/fpls.2019.00242

Bahin, E., Bailly, C., Sotta, B., Kranner, I., Corbineau, F., and Leymarie, J. (2011). Crosstalk between reactive oxygen species and hormonal signalling pathways regulates grain dormancy in barley. Plant Cell Environ. 34, 980-993. doi: 10. $1111 /$ j.1365-3040.2011.02298.x

Bailly, C. (2019). The signalling role of ROS in the regulation of seed germination and dormancy. Biochem. J. 476, 3019-3032. doi: 10.1042/BCJ20190159 that requires the careful regulation of external and internal biotic and abiotic interactions. The accurate and optimized coordination of phytohormones, light, temperature, heat shock proteins, ROS, and endosperm decay is required to permit testa rupture and subsequent germination. Seed germination therefore requires internal responsiveness to external environmental cues.

\section{AUTHOR CONTRIBUTIONS}

MF, XZ, and MZ wrote the manuscript. WM and JZ revised the manuscript. All authors contributed to the article and approved the submitted version.

\section{FUNDING}

This study was supported by the Innovative Research Group Project of Hebei Natural Science Foundation (Grant No. C2020204111), the National Natural Science Foundation of China (Grant Nos. 31801857 and 31930098), the Program for Young Talents of Hebei Education Department (BJ2021024), and the Natural Science Foundation of Hebei (C2019204201).

\section{ACKNOWLEDGMENTS}

We are thankful to Muhammad Haroon for providing valuable insights in the development of graphics for the manuscript.

Bailly, C., El-Maarouf-Bouteau, H., and Corbineau, F. (2008). From intracellular signaling networks to cell death: the dual role of reactive oxygen species in seed physiology. C. R. Biol. 331, 806-814. doi: 10.1016/j.crvi.2008.07.022

Barba-Espin, G., Nicolas, E., Almansa, M. S., Cantero-Navarro, E., Albacete, A., and Hernández, J. A. (2012). Role of thioproline on seed germination: interaction ROS-ABA and effects on antioxidative metabolism. Plant Physiol. Biochem. 59, 30-36. doi: 10.1016/j.plaphy.2011.12.002

Barrero, J. M., Talbot, M. J., White, R. G., Jacobsen, J. V., and Gubler, F. (2009). Anatomical and transcriptomic studies of the coleorhiza reveal the importance of this tissue in regulating dormancy in barley. Plant Physiol. 150, 1006-1021. doi: 10.1104/pp.109.137901

Beracochea, V. C., Almasia, N. I., Peluffo, L., Nahirñak, V., Hopp, E., and Paniego, N. (2015). Sunflower germin-like protein HaGLP1 promotes ROS accumulation and enhances protection against fungal pathogens in transgenic Arabidopsis thaliana. Plant Cell Rep. 34, 1717-1733. doi: 10.1007/s00299-015-1819-4

Best, C., Mizrahi, R., and Ostersetzer-Biran, O. (2020). Why so complex? the intricacy of genome structure and gene expression, associated with angiosperm mitochondria, may relate to the regulation of embryo quiescence or dormancy-intrinsic blocks to early plant life. Plants 9:598. doi: 10.3390/plants90 50598

Betekhtin, A., Milewska-Hendel, A., Lusinska, J., Chajec, L., Kurczynska, E., and Hasterok, R. (2018). Organ and tissue-specific localisation of selected cell wall epitopes in the zygotic embryo of Brachypodium distachyon. Int. J. Mol. Sci. 19:725. doi: 10.3390/ijms19030725

Bi, C., Ma, Y., Wu, Z., Yu, Y.-T., Liang, S., and Lu, K. (2017). Arabidopsis ABI5 plays a role in regulating ROS homeostasis by activating CATALASE 1 transcription in seed germination. Plant Mol. Biol. 94, 197-213. doi: 10.1007/ s11103-017-0603-y 
Bykova, N. V., Hoehn, B., Rampitsch, C., Banks, T., Stebbing, J. A., and Fan, T. (2011). Redox-sensitive proteome and antioxidant strategies in wheat seed dormancy control. Proteomics 11, 865-882. doi: 10.1002/pmic.200900810

Cai, F., Lan-Ju, M., Xiao-Long, A., Gao, S., Tang, L., and Chen, F. (2011). Lipid peroxidation and antioxidant responses during seed germination of Jatropha curcas. Int. J. Agric. Biol. 13, 25-30.

Carrera-Castaño, G., Calleja-Cabrera, J., Pernas, M., Gómez, L., and OñateSánchez, L. (2020). An updated overview on the regulation of seed germination. Plants 9:703. doi: 10.3390/plants9060703

Chateigner-Boutin, A. L., Ramos-Vega, M., Guevara-García, A., Andrés, C., de la Luz Gutiérrez-Nava, M., Cantero, A., et al. (2008). CLB19, a pentatricopeptide repeat protein required for editing of rpoA and clpP chloroplast transcripts. Plant J. 56, 590-602. doi: 10.1111/j.1365-313X.2008.03634.X

Chen, D., Li, Y., Fang, T., Shi, X., and Chen, X. (2016). Specific roles of tocopherols and tocotrienols in seed longevity and germination tolerance to abiotic stress in transgenic rice. Plant Sci. 244, 31-39. doi: 10.1016/j.plantsci.2015. 12.005

Cheng, S., Gutmann, B., Zhong, X., Ye, Y., Fisher, M. F., and Bai, F. (2016). Redefining the structural motifs that determine RNA binding and RNA editing by pentatricopeptide repeat proteins in land plants. Plant J. $85,532-547$. doi: $10.1111 /$ tpj.13121

Choquet, Y., and Wollman, F.-A. (2002). Translational regulations as specific traits of chloroplast gene expression. FEBS Lett. 529, 39-42. doi: 10.1016/s00145793(02)03260-x

Czarna, M., Kolodziejczak, M., and Janska, H. (2016). Mitochondrial proteome studies in seeds during germination. Proteomes 4:19. doi: 10.3390/proteomes4020019

Dai, D., Luan, S., Chen, X., Wang, Q., Feng, Y., Zhu, C., et al. (2018). Maize Dek37 encodes a P-type PPR protein that affects cis-splicing of mitochondrial nad2 intron 1 and seed development. Genetics 208, 1069-1082. doi: 10.1534/genetics. 117.300602

De Longevialle, A. F., Hendrickson, L., Taylor, N. L., Delannoy, E., Lurin, C., and Badger, M. (2008). The pentatricopeptide repeat gene OTP51 with two LAGLIDADG motifs is required for the cis-splicing of plastid ycf3 intron 2 in Arabidopsis thaliana. Plant J. 56, 157-168. doi: 10.1111/j.1365-313X.2008. 03581.x

Dębska, K., Krasuska, U., Budnicka, K., Bogatek, R., and Gniazdowska, A. (2013). Dormancy removal of apple seeds by cold stratification is associated with fluctuation in $\mathrm{H} 2 \mathrm{O} 2$. NO production and protein carbonylation level. J. Plant Physiol. 170, 480-488. doi: 10.1016/j.jplph.2012.11.018

di Donato, M., and Geisler, M. (2019). HSP 90 and co-chaperones, a multitaskers' view on plant hormone biology. FEBS Lett. 593, 1415-1430. doi: 10.1002/18733468.13499

Ding, Y., Shi, Y., and Yang, S. (2020). Molecular regulation of plant responses to environmental temperatures. Mol. Plant. 13, 544-564. doi: 10.1016/j.molp. 2020.02.004

Ding, Y.-H., Liu, N.-Y., Tang, Z.-S., Liu, J., and Yang, W. (2006). Arabidopsis GLUTAMINE-RICH PROTEIN23 is essential for early embryogenesis and encodes a novel nuclear PPR motif protein that interacts with RNA polymerase II subunit III. Plant Cell 18, 815-830. doi: 10.1105/tpc.105.039495

El-Maarouf-Bouteau, H., and Bailly, C. (2008). Oxidative signaling in seed germination and dormancy. Plant Signal. Behav. 3, 175-182. doi: 10.4161/psb. 3.3.5539

El-Maarouf-Bouteau, H., Sajjad, Y., Bazin, J., Langlade, N., Cristescu, S. M., and Balzergue, S. (2015). Reactive oxygen species, abscisic acid and ethylene interact to regulate sunflower seed germination. Plant Cell and Environ. 38, 364-374. doi: $10.1111 /$ pce. 12371

Essemine, J., Ammar, S., and Bouzid, S. (2010). Impact of heat stress on germination and growth in higher plants, physiological, biochemical and molecular repercussions and mechanisms of defence. J. Biol. Sci. 10, 565-572. doi: $10.3923 /$ jbs.2010.565.572

Foyer, C. H., Kyndt, T., and Hancock, R. (2020). Vitamin C in plants: novel concepts, new perspectives, and outstanding issues. Antioxid. Redox. Signal. 32, 463-485. doi: 10.1089/ars.2019.7819

Galland, M., Huguet, R., Arc, E., Cueff, G., Job, D., and Rajjou, L. (2014). Dynamic proteomics emphasizes the importance of selective mRNA translation and protein turnover during Arabidopsis seed germination. Mol. Cell Proteomics 13, 252-268. doi: 10.1074/mcp.M113.032227
Gomes, M., and Garcia, Q. (2013). Reactive oxygen species and seed germination. Biologia 68, 351-357.

Gomes, M., Carneiro, M., Nogueira, C., Soares, A., and Garcia, Q. (2013). The system modulating ROS content in germinating seeds of two Brazilian savanna tree species exposed to As and Zn. Acta Physiol. Plant. 35, 1011-1022. doi: 10.1007/s11738-012-1140-6

Gong, F., Yao, Z., Liu, Y., Sun, M., and Peng, X. (2021). H2O2 response gene 1/2 are novel sensors or responders of $\mathrm{H} 2 \mathrm{O} 2$ and involve in maintaining embryonic root meristem activity in Arabidopsis thaliana. Plant Sci. 310:110981.

González-Calle, V., Barrero-Sicilia, C., Carbonero, P., and Iglesias-Fernández, R. (2015). Mannans and endo- $\beta$-mannanases (MAN) in Brachypodium distachyon: expression profiling and possible role of the BdMAN genes during coleorhizalimited seed germination. J. Exp. Bot. 66, 3753-3764. doi: 10.1093/jxb/erv168

Hajihashemi, S., Skalicky, M., Brestic, M., and Pavla, V. (2020). Cross-talk between nitric oxide, hydrogen peroxide and calcium in salt-stressed Chenopodium quinoa Willd. at seed germination stage. Plant Physiol. Biochem. 154, 657-664. doi: 10.1016/j.plaphy.2020.07.022

Hao, Y., Wang, Y., Wu, M., Zhu, X., Teng, X., and Sun, Y. (2019). The nuclearlocalized PPR protein OsNPPR1 is important for mitochondrial function and endosperm development in rice. J. Exp. Bot. 70, 4705-4720. doi: 10.1093/jxb/ erz226

He, H., Willems, L. A., Batushansky, A., Fait, A., Hanson, J., and Nijveen, H. (2016). Effects of parental temperature and nitrate on seed performance are reflected by partly overlapping genetic and metabolic pathways. Plant Cell Physiol. 57, 473-487. doi: 10.1093/pcp/pcv207

He, J., Duan, Y., Hua, D., Fan, G., Wang, L., and Liu, Y. (2012). DEXH box RNA helicase-mediated mitochondrial reactive oxygen species production in Arabidopsis mediates crosstalk between abscisic acid and auxin signaling. Plant Cell 24, 1815-1833. doi: 10.1105/tpc.112.098707

He, Y., Ye, Z., Ying, Q., Ma, Y., Zang, Y., and Wang, H. (2019). Glyoxylate cycle and reactive oxygen species metabolism are involved in the improvement of seed vigor in watermelon by exogenous GA3. Sci. Hortic. 247, 184-194.

Henriet, C., Balliau, T., Aimé, D., Le Signor, C., Kreplak, J., and Zivy, M. (2021). Proteomics of developing pea seeds reveals a complex antioxidant network underlying the response to sulfur deficiency and water stress. J. Exp. Bot. 72, 2611-2626. doi: 10.1093/jxb/eraa571

Holloway, T., Steinbrecher, T., Pérez, M., Seville, A., Stock, D., and Nakabayashi, K. (2021). Coleorhiza-enforced seed dormancy: a novel mechanism to control germination in grasses. New Phytol. 229, 2179-2191. doi: 10.1111/nph.16948

Howell, K. A., Narsai, R., Carroll, A., Ivanova, A., Lohse, M., and Usadel, B. (2009). Mapping metabolic and transcript temporal switches during germination in rice highlights specific transcription factors and the role of RNA instability in the germination process. Plant Physiol. 149, 961-980. doi: 10.1104/pp.108.129874

Iglesias-Fernández, R., Pastor-Mora, E., Vicente-Carbajosa, J., and Carbonero, P. (2020). A possible role of the aleurone expressed gene HvMAN1 in the hydrolysis of the cell wall mannans of the starchy endosperm in germinating Hordeum vulgare L. seeds. Front. Plant Sci. 10:1706. doi: 10.3389/fpls.2019. 01706

Ishibashi, Y., Aoki, N., Kasa, S., Sakamoto, M., Kai, K., and Tomokiyo, R. (2017). The interrelationship between abscisic acid and reactive oxygen species plays a key role in barley seed dormancy and germination. Front. Plant Sci. 8:275. doi: 10.3389/fpls.2017.00275

Ishibashi, Y., Kasa, S., Sakamoto, M., Aoki, N., Kai, K., and Yuasa, T. (2015). A role for reactive oxygen species produced by NADPH oxidases in the embryo and aleurone cells in barley seed germination. PLoS One 10:e0143173. doi: 10.1371/journal.pone.0143173

Ishibashi, Y., Koda, Y., Zheng, S.-H., Yuasa, T., and Iwaya-Inoue, M. (2013). Regulation of soybean seed germination through ethylene production in response to reactive oxygen species. Ann. Bot. 111, 95-102. doi: 10.1093/aob/ $\operatorname{mcs} 240$

Jänsch, L., Kruft, V., Schmitz, U. K., and Braun, H. (1996). New insights into the composition, molecular mass and stoichiometry of the protein complexes of plant mitochondria. Plant J. 9, 357-368. doi: 10.1046/j.1365-313x.1996. 09030357.x

Jones, R. (1974). The structure of the lettuce endosperm. Planta 121, 133-146. doi: $10.1007 / \mathrm{BF} 00388752$

Kai, K., Kasa, S., Sakamoto, M., Aoki, N., Watabe, G., and Yuasa, T. (2016). Role of reactive oxygen species produced by NADPH oxidase in gibberellin 
biosynthesis during barley seed germination. Plant Signal. Behav. 11:e1180492. doi: 10.1080/15592324.2016.1180492

Kaur, H., Petla, B. P., and Majee, M. (2016). "Small heat shock proteins, roles in development, desiccation tolerance and seed longevity," in Heat Shock Proteins and Plants. Heat Shock Proteins, Vol 10, eds A. Asea, P. Kaur, and S. Calderwood (Cham: Springer). doi: 10.1093/jxb/erv244

Kranner, I., Roach, T., Beckett, R. P., Whitaker, C., and Minibayeva, F. (2010). Extracellular production of reactive oxygen species during seed germination and early seedling growth in Pisum sativum. J. Plant Physiol. 167, 805-811. doi: $10.1016 /$ j.jplph.2010.01.019

Krasuska, U., Ciacka, K., Bogatek, R., and Gniazdowska, A. (2014). Polyamines and nitric oxide link in regulation of dormancy removal and germination of apple (Malus domestica Borkh.) embryos. J. Plant Growth Regul. 33, 590-601. doi: $10.1007 /$ s00344-013-9408-7

Lariguet, P., Ranocha, P., De Meyer, M., Barbier, O., Penel, C., and Dunand, C. (2013). Identification of a hydrogen peroxide signalling pathway in the control of light-dependent germination in Arabidopsis. Planta 238, 381-395. doi: 10.1007/s00425-013-1901-5

Law, S. R., Narsai, R., and Whelan, J. (2014). Mitochondrial biogenesis in plants during seed germination. Mitochondrion 19, 214-221. doi: 10.1016/j.mito.2014. 04.002

Law, S. R., Narsai, R., Taylor, N. L., Delannoy, E., Carrie, C., and Giraud, E. (2012). Nucleotide and RNA metabolism prime translational initiation in the earliest events of mitochondrial biogenesis during Arabidopsis germination. Plant Physiol. 158, 1610-1627. doi: 10.1104/pp.111.192351

Leymarie, J., Vitkauskaité, G., Hoang, H. H., Gendreau, E., Chazoule, V., and Meimoun, P. (2012). Role of reactive oxygen species in the regulation of Arabidopsis seed dormancy. Plant Cell Physiol. 53, 96-106. doi: 10.1093/pcp/ pcr129

Li, L., Nelson, C. J., Solheim, C., Whelan, J., and Millar, A. H. (2012). Determining degradation and synthesis rates of Arabidopsis proteins using the kinetics of progressive $15 \mathrm{~N}$ labeling of two-dimensional gel-separated protein spots. Mol. Cell Proteomics 11:M111.010025. doi: 10.1074/mcp.M111.010025

Li, W.-Y., Chen, B.-X., Chen, Z.-J., Gao, Y.-T., Chen, Z., and Liu, J. (2017). Reactive oxygen species generated by NADPH oxidases promote radicle protrusion and root elongation during rice seed germination. Int. J. Mol. Sci. 18:110. doi: 10.3390/ijms 18010110

Lin, Y., Yang, L., Paul, M., Zu, Y., and Tang, Z. (2013). Ethylene promotes germination of Arabidopsis seed under salinity by decreasing reactive oxygen species: evidence for the involvement of nitric oxide simulated by sodium nitroprusside. Plant Physiol. Biochem. 73, 211-218. doi: 10.1016/j.plaphy.2013. 10.003

Liu, J., Hasanuzzaman, M., Wen, H., Zhang, J., Peng, T., and Sun, H. (2019). High temperature and drought stress cause abscisic acid and reactive oxygen species accumulation and suppress seed germination growth in rice. Protoplasma 256, 1217-1227. doi: 10.1007/s00709-019-01354-6

Liu, X., and Hou, X. (2018). Antagonistic regulation of ABA and GA in metabolism and signaling pathways. Front. Plant Sci. 9:251. doi: 10.3389/fpls.2018.00251

Liu, Y., Ye, N., Liu, R., Chen, M., and Zhang, J. (2010). H2O2 mediates the regulation of ABA catabolism and GA biosynthesis in Arabidopsis seed dormancy and germination. J. Exp. Bot. 61, 2979-2990. doi: 10.1093/jxb/erq125

Logan, D. C., Millar, A. H., Sweetlove, L. J., Hill, S. A., and Leaver, C. (2001). Mitochondrial biogenesis during germination in maize embryos. Plant Phsyiol. 125, 662-672. doi: 10.1104/pp.125.2.662

Ma, W., Guan, X., Li, J., Pan, R., Wang, L., and Liu, F. (2019). Mitochondrial small heat shock protein mediates seed germination via thermal sensing. Proc. Natl. Acad. Sci. 116, 4716-4721. doi: 10.1073/pnas.1815790116

Ma, W., Zhao, T., Li, J., Liu, B., Fang, L., and Hu, Y. (2016). Identification and characterization of the GhHsp20 gene family in Gossypium hirsutum. Sci. Rep. 6:32517. doi: 10.1038/srep32517

Ma, Z., Bykova, N. V., and Igamberdiev, A. (2017). Cell signaling mechanisms and metabolic regulation of germination and dormancy in barley seeds. Crop J. 5 , 459-477.

Ma, Z., Marsolais, F., Bykova, N. V., and Igamberdiev, A. (2016). Nitric oxide and reactive oxygen species mediate metabolic changes in barley seed embryo during germination. Front. Plant Sci. 7:138. doi: 10.3389/fpls.2016.00138

Meng, Y., Shuai, H., Luo, X., Chen, F., Zhou, W., Yang, W., et al. (2017). Karrikins: regulators involved in phytohormone signaling networks during seed germination and seedling development. Front. Plant Sci. 7:2021. doi: 10.3389/ fpls.2016.02021

Morscher, F., Kranner, I., Arc, E., Bailly, C., and Roach, T. (2015). Glutathione redox state, tocochromanols, fatty acids, antioxidant enzymes and protein carbonylation in sunflower seed embryos associated with after-ripening and ageing. Ann. Bot. 116, 669-678. doi: 10.1093/aob/mcv108

Murgia, I., and Morandini, P. (2017). Iron deficiency prolongs seed dormancy in Arabidopsis plants. Front. Plant Sci. 8:2077. doi: 10.3389/fpls.2017.02077

Nakamura, T., Meierhoff, K., Westhoff, P., and Schuster, G. (2003). RNA-binding properties of HCF152, an Arabidopsis PPR protein involved in the processing of chloroplast RNA. Eur. J. Biochem. 270, 4070-4081. doi: 10.1046/j.1432-1033. 2003.03796.x

Narsai, R., Law, S. R., Carrie, C., Xu, L., and Whelan, J. (2011). In-depth temporal transcriptome profiling reveals a crucial developmental switch with roles for RNA processing and organelle metabolism that are essential for germination in Arabidopsis. Plant Physiol. 157, 1342-1362. doi: 10.1104/pp.111. 183129

Nourimand, M., and Todd, C. (2019). There is a direct link between allantoin concentration and cadmium tolerance in Arabidopsis. Plant Physiol. Biochem. 135, 441-449. doi: 10.1016/j.plaphy.2018.11.016

Oh, E., Kim, J., Park, E., Kim, J.-I., Kang, C., and Choi, G. (2004). PIL5, a phytochrome-interacting basic helix-loop-helix protein, is a key negative regulator of seed germination in Arabidopsis thaliana. Plant Cell 16, 3045-3058. doi: $10.1105 /$ tpc. 104.025163

Oracz, K., and Karpiński, S. (2016). Phytohormones signaling pathways and ROS involvement in seed germination. Front. Plant Sci. 7:864. doi: 10.3389/fpls.2016. 00864

Oracz, K., Bouteau, H. E. M., Farrant, J. M., Cooper, K., Belghazi, M., and Job, C. (2007). ROS production and protein oxidation as a novel mechanism for seed dormancy alleviation. Plant J. 50, 452-465. doi: 10.1111/j.1365-313X.2007. 03063.x

Oracz, K., El-Maarouf-Bouteau, H., Kranner, I., Bogatek, R., Corbineau, F., and Bailly, C. (2009). The mechanisms involved in seed dormancy alleviation by hydrogen cyanide unravel the role of reactive oxygen species as key factors of cellular signaling during germination. Plant Physiol. 150, 494-505. doi: 10.1104/ pp.109.138107

Ortiz-Espín, A., Iglesias-Fernández, R., Calderón, A., Carbonero, P., Sevilla, F., and Jiménez, A. (2017). Mitochondrial AtTrxo1 is transcriptionally regulated by AtbZIP9 and AtAZF2 and affects seed germination under saline conditions. J. Exp. Bot. 68, 1025-1038. doi: 10.1093/jxb/erx012

Qi, W., Tian, Z., Lu, L., Chen, X., Chen, X., and Zhang, W. (2017). Editing of mitochondrial transcripts nad 3 and cox 2 by Dek10 is essential for mitochondrial function and maize plant development. Genetics 205, 1489-1501. doi: 10.1534/genetics.116.199331

Rao, R., Salvato, F., Thal, B., Eubel, H., Thelen, J., and Møller, I. (2017). The proteome of higher plant mitochondria. Mitochondrion 33, 22-37. doi: 10.1016/ j.mito.2016.07.002

Scharf, K.-D., Siddique, M., and Vierling, E. (2001). The expanding family of Arabidopsis thaliana small heat stress proteins and a new family of proteins containing $\alpha$-crystallin domains (Acd proteins). Cell Stress Chaperones 6:225. doi: 10.1379/1466-1268(2001)006<0225:tefoat $>2.0 . c 0 ; 2$

Shu, K., Liu, X.-D., Xie, Q., and He, Z.-H. (2016). Two faces of one seed, hormonal regulation of dormancy and germination. Mol. Plant 9, 34-45. doi: 10.1016/j. molp.2015.08.010

Shuai, H., Meng, Y., Luo, X., Chen, F., Zhou, W., and Dai, Y. (2017). Exogenous auxin represses soybean seed germination through decreasing the gibberellin/abscisic acid (GA/ABA) ratio. Sci. Rep. 7:12620. doi: 10.1038/ s41598-017-13093-w

Singh, K. L., Chaudhuri, A., and Kar, R. (2015). Role of peroxidase activity and $\mathrm{Ca} 2+$ in axis growth during seed germination. Planta 242, 997-1007. doi: 10. 1007/s00425-015-2338-9

Small, I., Peeters, N., Legeai, F., and Lurin, C. (2004). Predotar: a tool for rapidly screening proteomes for $\mathrm{N}$-terminal targeting sequences. Proteomics 4, 15811590. doi: 10.1002/pmic. 200300776

Stawska, M., and Oracz, K. (2019). PhyB and HY5 are involved in the blue light-mediated alleviation of dormancy of Arabidopsis seeds possibly via the modulation of expression of genes related to light, GA, and ABA. Int. J. Mol. Sci. 20:5882. doi: $10.3390 /$ ijms 20235882 
Su, L., Lan, Q., Pritchard, H. W., Xue, H., and Wang, X. (2016). Reactive oxygen species induced by cold stratification promote germination of Hedysarum scoparium seeds. Plant Physiol. Biochem. 109, 406-415. doi: 10.1016/j.plaphy. 2016.10.025

Sun, F., Xiu, Z., Jiang, R., Liu, Y., Zhang, X., and Yang, Y.-Z. (2019). The mitochondrial pentatricopeptide repeat protein EMP12 is involved in the splicing of three nad2 introns and seed development in maize. J. Exp. Bot. 70, 963-972. doi: 10.1093/jxb/ery432

Sun, L. R., Zhao, Z. J., and Hao, F. (2019). NADPH oxidases, essential players of hormone signalings in plant development and response to stresses. Plant Signal. Behav. 14:1657343. doi: 10.1080/15592324.2019.1657343

Taylor, N. L., Howell, K. A., Heazlewood, J. L., Tan, T. Y. W., Narsai, R., Huang, S., et al. (2010). Analysis of the rice mitochondrial carrier family reveals anaerobic accumulation of a basic amino acid carrier involved in arginine metabolism during seed germination. Plant Physiol. 154, 691-704. doi: 10.1104/pp.110. 162214

Visscher, A. M., Yeo, M., Barreiro, P. G., Stuppy, W., Frances, A. L., Di, S. A., et al. (2018). Dry heat exposure increases hydrogen peroxide levels and breaks physiological seed coat-imposed dormancy in Mesembryanthemum crystallinum (Aizoaceae) seeds. Env. Exp. Bot. 155, 272-280. doi: 10.1016/j. envexpbot.2018.07.009

Wagner, S., Van, A. O., Elsässer, M., and Schwarzländer, M. (2018). Mitochondrial energy signaling and its role in the low-oxygen stress response of plants. Plant Physiol. 176, 1156-1170. doi: 10.1104/pp.17.01387

Wang, H., Zhao, K., Li, X., Chen, X., Liu, W., and Wang, J. (2020). Factors affecting seed germination and emergence of Aegilops tauschii. Weed Res. 60, 171-181. doi: $10.1111 /$ wre. 12410

Wang, Y., Htwe, Y. M., Li, J., Shi, P., Zhang, D., Zhao, Z., et al. (2019). Integrative omics analysis on phytohormones involved in oil palm seed germination. $B M C$ Plant Biol. 19:363. doi: 10.1186/s12870-019-1970-0

Wehmeyer, N., and Vierling, E. (2000). The expression of small heat shock proteins in seeds responds to discrete developmental signals and suggests a general protective role in desiccation tolerance. Plant Physiol. 122, 1099-1108. doi: 10.1104/pp.122.4.1099

Whitaker, C., Beckett, R., Minibayeva, F., and Kranner, I. (2010). Alleviation of dormancy by reactive oxygen species in Bidens pilosa L. seeds. S. Afr. J. Bot. 76, 601-605.

Xia, Q., Ponnaiah, M., Cueff, G., Rajjou, L., Prodhomme, D., and Gibon, Y. (2018). Integrating proteomics and enzymatic profiling to decipher seed metabolism affected by temperature in seed dormancy and germination. Plant Sci. 269, $118-125$.

Xiu, Z., Sun, F., Shen, Y., Zhang, X., Jiang, R., Bonnard, G., et al. (2016). EMPTY PERICARP 16 is required for mitochondrial nad 2 intron 4 cis-splicing, complex I assembly and seed development in maize. Plant J. 85, 507-519. doi: 10.1111/ tpj. 13122

Yamazaki, H., Tasaka, M., and Shikanai, T. (2004). PPR motifs of the nucleusencoded factor, PGR3, function in the selective and distinct steps of chloroplast gene expression in Arabidopsis. Plant J. 38, 152-163. doi: 10.1111/j.1365-313X. 2004.02035.x

Yang, L., Wang, X., Chang, N., Nan, W., Wang, S., Ruan, M., et al. (2019b). Cytosolic glucose-6-phosphate dehydrogenase is involved in seed germination and root growth under salinity in Arabidopsis. Front. Plant Sci. 10:182. doi: 10.3389/fpls.2019.00182

Yang, L., Wang, S., Sun, L., Ruan, M., Li, S., He, R., et al. (2019a). Involvement of G6PD5 in ABA response during seed germination and root growth in Arabidopsis. BMC Plant Biol. 19:44. doi: 10.1186/s12870-019-1647-8
Yang, X., He, K., Chi, X., Chai, G., Wang, Y., Jia, C., et al. (2018). Miscanthus NAC transcription factor MlNAC12 positively mediates abiotic stress tolerance in transgenic Arabidopsis. Plant Sci. 277, 229-241. doi: 10.1016/j.plantsci.2018. 09.013

Yang, X., Zhang, F., Yang, M., He, Y., Li, Z., Yang, J., et al. (2020). The NADPHoxidase LsRbohC1 plays a role in lettuce (Lactuca sativa) seed germination. Plant Physiol. Biochem. 154, 751-757. doi: 10.1016/j.plaphy.2020.05.042

Ye, N., and Zhang, J. (2012). Antagonism between abscisic acid and gibberellins is partially mediated by ascorbic acid during seed germination in rice. Plant Signal. Behav. 7, 563-565. doi: 10.4161/psb.19919

Zhang, H., He, D., Li, M., and Yang, P. (2017). Carbonylated protein changes between active germinated embryos and quiescent embryos give insights into rice seed germination regulation. Plant Growth Regul. 83, 335-350.

Zhang, K., Yao, L., Zhang, Y., Baskin, J. M., Baskin, C. C., and Xiong, Z. (2019). A review of the seed biology of Paeonia species (Paeoniaceae), with particular reference to dormancy and germination. Planta 249, 291-303. doi: 10.1007/ s00425-018-3017-4

Zhang, T., Hu, Y., Jiang, W., Fang, L., Guan, X., Chen, J., et al. (2015). Sequencing of allotetraploid cotton (Gossypium hirsutum L. acc. TM-1) provides a resource for fiber improvement. Nat. Biotechnol. 33, 531-537. doi: 10.1038/nbt.3207

Zhang, Y., Chen, B., Xu, Z., Shi, Z., Chen, S., and Huang, X. (2014). Involvement of reactive oxygen species in endosperm cap weakening and embryo elongation growth during lettuce seed germination. J. Exp. Bot. 65, 3189-3200. doi: 10. 1093/jxb/eru167

Zhang, Y., Shi, H., and Deng, B. (2018). Mutagen-induced phytotoxicity in maize seed germination is dependent on ROS scavenging capacity. Sci. Rep. 8:14078. doi: 10.1038/s41598-018-32271-y

Zhao, Y., Luo, L., Xu, J., Xin, P., Guo, H., and Wu, J. (2018). Malate transported from chloroplast to mitochondrion triggers production of ROS and PCD in Arabidopsis thaliana. Cell Res. 28, 448-461. doi: 10.1038/s41422-018-0024-8

Zhou, L., Ye, Y., Zhao, Q., Du, X., Zakari, S. A., and Su, D. (2018). Suppression of ROS generation mediated by higher InsP 3 level is critical for the delay of seed germination in lpa rice. Plant Growth Regul. 85, 411-424. doi: 10.1007/s10725018-0402-8

Zhou, Y., Chen, H., Chu, P., Li, Y., Tan, B., and Ding, Y. (2012). NnHSP17.5, a cytosolic class II small heat shock protein gene from Nelumbo nucifera, contributes to seed germination vigor and seedling thermotolerance in transgenic Arabidopsis. Plant Cell Rep. 31, 379-389. doi: 10.1007/s00299-011$1173-0$

Conflict of Interest: The authors declare that the research was conducted in the absence of any commercial or financial relationships that could be construed as a potential conflict of interest.

Publisher's Note: All claims expressed in this article are solely those of the authors and do not necessarily represent those of their affiliated organizations, or those of the publisher, the editors and the reviewers. Any product that may be evaluated in this article, or claim that may be made by its manufacturer, is not guaranteed or endorsed by the publisher.

Copyright (๑) 2021 Farooq, Zhang, Zafar, Ma and Zhao. This is an open-access article distributed under the terms of the Creative Commons Attribution License (CC BY). The use, distribution or reproduction in other forums is permitted, provided the original author(s) and the copyright owner(s) are credited and that the original publication in this journal is cited, in accordance with accepted academic practice. No use, distribution or reproduction is permitted which does not comply with these terms. 